\title{
The efficiency of micrometastasis by sentinel node navigation surgery using indocyanine green and infrared ray laparoscopy system for gastric cancer
}

\author{
Kentaro Yano $\cdot$ Hiroshi Nimura $\cdot$ Norio Mitsumori • \\ Naoto Takahashi • Hideyuki Kashiwagi • \\ Katsuhiko Yanaga
}

Received: 4 May 2011/Accepted: 23 September 2011/Published online: 27 October 2011

(C) The International Gastric Cancer Association and The Japanese Gastric Cancer Association 2011

\begin{abstract}
Background The clinical application of sentinel node navigation surgery (SNNS) for patients with gastric cancer requires accurate intraoperative diagnosis of lymph node metastasis. However, the clinical significance of the diagnosis of lymph node micrometastasis for gastric cancer has not been established. In this study, we evaluated lymph nodes dissected during SNNS by immunohistochemistry with anti-cytokeratin antibody (IHC) staining for gastric cancer to investigate the usefulness of SNNS.

Patients and methods The subjects were 130 patients with gastric cancer (3,381 lymph nodes) who underwent SNNS with infrared ray observation and lymph node dissection of $\mathrm{D} 1+\alpha$ or more. The dissected lymph nodes were stained with IHC (CAM 5.2), and the results were compared with intra- and postoperative diagnoses by hematoxylin and eosin (H\&E) staining. In addition, the association of metastatic lymph nodes and ICG-positive lymph nodes was examined.

Results The number of patients (lymph nodes) with lymph node metastasis by HE and IHC staining was 16 (52 nodes) and 31 (91 nodes), respectively. Fifteen patients (27 nodes) diagnosed with pNO by HE staining were diagnosed to be metastatic by IHC staining. The tumor depth of these patients was pT1 in ten patients $(\mathrm{m}, 3 ; \mathrm{sm}, 7)$ and $\mathrm{pT} 2$ in
\end{abstract}

We confirm that this manuscript has not been published or submitted for publication elsewhere except as a brief abstract in the proceedings of a scientific meeting or symposium.

K. Yano $(\bowtie) \cdot$ H. Nimura · N. Mitsumori - N. Takahashi ·

H. Kashiwagi · K. Yanaga

Department of Surgery, The Jikei University School

of Medicine, 3-25-8 Nishi-shinbashi, Minato-ku,

Tokyo 105-8461, Japan

e-mail: yanoken@jikei.ac.jp five (mp, 4; ss, 1). Regarding the histological type, three patients were classified as well-differentiated type, while six patients each had moderately and poorly differentiated types. The grade of lymphatic invasion was ly0 in 5, ly1 in 6 , and ly 2 in 4, respectively. Histological assessment of 27 IHC-positive and HE-negative cells indicated 5 single cells, 16 clusters, and 6 micrometastases. These lymph nodes were all included in the sentinel nodes (SN) identified during surgery. All but one patient $(0.8 \%)$ were recurrence-free at 2-8 years after surgery (median 74.7 months). The one patient developed anastomotic recurrence 4.5 years after the first operation and died.

Conclusion Since all 27 lymph nodes in 15 patients with metastasis by IHC staining but not by HE staining were micrometastasis or less and included in the SN, ICGpositive lymphatic basin dissection by SNNS with infrared ray observation seems to be an adequate method of lymph node dissection for gastric cancer.

Keywords Sentinel node navigation surgery - Infrared ray · Micrometastasis · Cytokerartin · Gastric cancer

\section{Introduction}

In Japan, D2 lymph node dissection in gastric cancer surgery is a standard technique for advanced gastric cancer to achieve acceptable long-term outcomes [1]. Recently, however, partial lymph node dissection and limited surgery for early gastric cancer have been described in the guidelines from the Japanese Gastric Cancer Association [2]. Among early gastric cancers, intramucosal carcinoma has been shown to exhibit minimal change of lymph node metastasis, for which endoscopic mucosal resection (EMR) and endoscopic submucosal dissection (ESD) are commonly 
performed [3]. Although approximately $20 \%$ of cancers clinically diagnosed as intramucosal carcinoma have been confirmed to invade the submucosa (SM) or more by pathological diagnosis [4], Gotoda et al. [5] showed that no lymph node metastasis was evident in gastric cancers that are SM1, differentiated carcinoma without lymphatic invasion, and $3 \mathrm{~cm}$ or less in size. Since patients without such factors or those who have the possibility for lymph node metastasis based on pathological findings diagnosed after EMR or ESD are treated with salvage surgery associated with lymph node dissection, it should be reasonable to establish to what extent lymph node dissection should be performed. One of these approaches is the sentinel node (SN) concept, which has recently attracted attention in malignant melanoma and breast cancer [6, 7].

Many clinical studies on SN for gastric cancer have been conducted in Japan and have shown good results [8-23]. We invented an infrared ray observation method for SN identification and performed sentinel node navigation surgery (SNNS) $[17,20]$. However, this approach cannot be applied in practice without accurate intraoperative metastatic diagnosis of the identified SN. Accurate intraoperative diagnosis of lymph node metastasis is very important for developing the strategy to perform minimally invasive surgery. Thin slice and immunostaining are considered ideal for rapid intraoperative diagnosis and are feasible in many institutions these days, but we mostly had to choose hematoxylin and eosin (H\&E) staining of frozen sections. We examined all of the lymph nodes dissected in SNNS stained with $\mathrm{H} \& \mathrm{E}$ as well as immunohistochemistry with anti-cytokeratin antibody (IHC) to investigate whether intraoperative diagnosis was possible and whether clinical application of SNNS by infrared ray observation is feasible.

\section{Patients and methods}

The subjects were 130 patients with gastric cancer clinically diagnosed as T1(109 patients, $84 \%)$ or T2 (21 patients, 16\%) and N0 who underwent SNNS with infrared ray observation and lymph node dissection of D $1+\alpha$ or more from July 2000 to August 2006. As for lymph node dissection, we performed $\mathrm{D} 1+\alpha$ or $\beta$ for $\mathrm{cT} 1$ and D2 for cT2, following the guidelines from the Japanese Gastric Cancer Association [2]. The definite pathological examination included $\mathrm{H} \& \mathrm{E}$ staining as well as IHC on one slice per node, taken from the mid-portion of the node (CAM 5.2; Becton Dickinson, San Jose, CA, USA). To identify the SN, $0.5 \mathrm{ml}$ of a $0.5-\mathrm{mg} / \mathrm{ml}$ indocyanine green (ICG; Diagnogreen, Daiichi Sankyo Co., Ltd., Japan) was injected endoscopically at four points around the cancer after laparotomy or insertion of a laparoscope under general anesthesia, and ICG-stained lymphatic vessels and nodes were observed using infrared ray electronic endoscopy (IREE, Olympus Optical, Tokyo, Japan) 20 min later. All lymph nodes stained during the specified time were defined as ICG-positive lymph nodes and considered to be SN. In general, lymphatic basin dissection (LBD) [11] was performed, and the SN was examined by intraoperative diagnosis of a frozen section. In patients treated with LBD, SN identification of other lymphatic basins was not performed after surgery. In patients not treated by LBD, SN identification of intraoperative ICG-positive lymphatic basins was performed after surgery. For diagnosis of lymph node metastasis, all dissected lymph nodes were first stained with HE, followed by IHC on the consecutive slice. In the diagnosis of metastasis by IHC staining, IHC-positive cells were classified as macrometastases $(>2 \mathrm{~mm})$, micrometastases $(0.2-2 \mathrm{~mm})$, or isolated tumor cells (ITC $<0.2 \mathrm{~mm}$ ) [24]. ITCs were further classified into two subcategories, single cell and cluster. Pathological characteristics of patients diagnosed as having metastases by IHC staining but not by HE staining were examined.

\section{Results}

The sentinel node was identified in all 130 patients (100\%). The sensitivities of HE and IHC staining were both $100 \%$ (Table 1).

Of 130 patients, lymph node metastasis was detected by $\mathrm{HE}$ and IHC staining in $16(12 \%)$ and 31 (24\%), respectively. Of 114 patients who did not have lymph node metastases by HE staining, 15 (13\%) were diagnosed with lymph node metastasis by IHC staining. Of 3,381 lymph nodes, $52(2 \%)$ and 91 (3\%) were diagnosed as metastatic nodes by HE and IHC staining, respectively (Table 2). No lymph nodes diagnosed positive for metastasis by $\mathrm{HE}$ staining were negative by IHC staining.

The pathological characteristics of the 15 patients diagnosed with pNO by HE staining but with pathological

Table 1 Sentinel node identification rate and sensitivity with infrared ray observation

\begin{tabular}{lll}
\hline SN identification rate & \multicolumn{2}{l}{ Sensitivity } \\
\cline { 2 - 3 } & HE & IHC \\
\hline $130 / 130$ & $16 / 16$ & $31 / 31$ \\
$(100 \%)$ & $(100 \%)$ & $(100 \%)$ \\
\hline
\end{tabular}

$\overline{H E \text { Hematoxylin and eosin staining, } I H C \text { immunohistochemistry with }}$ anti-cytokeratin antibody staining 
Table 2 Diagnosis of lymph node metastasis stained by HE and IHC

\begin{tabular}{lll}
\hline & \multicolumn{2}{l}{ Positive staining } \\
\cline { 2 - 3 } & HE & IHC \\
\hline Patients $(n=130)$ & $16(12 \%)$ & $31(24 \%)$ \\
Lymph nodes $(n=3,381)$ & $52(2 \%)$ & $91(3 \%)$ \\
\hline
\end{tabular}

Fifteen patients ( 27 nodes) diagnosed as pNO by HE staining were judged to be metastatic by IHC staining

$H E$ Hematoxylin and eosin staining, $I H C$ immunohistochemistry with anti-cytokeratin antibody staining

Table 3 Pathological characteristics of the 15 patients diagnosed as pNO by HE staining but $\mathrm{pN}+$ by IHC

\begin{tabular}{|c|c|c|c|c|}
\hline \multirow[t]{5}{*}{ Depth $^{a}$} & $\mathrm{M}$ & 3 & SM2 & 6 \\
\hline & SM1 & 1 & MP & 4 \\
\hline & & & SS & 1 \\
\hline & & & SE & 0 \\
\hline & & & $4(27 \%)$ & $11(73 \%)$ \\
\hline \multirow[t]{3}{*}{ Histological type ${ }^{b}$} & Well & 3 & Moderate & 6 \\
\hline & & & Poor & 6 \\
\hline & & $3(20 \%)$ & & $12(80 \%)$ \\
\hline \multirow[t]{4}{*}{ Lymphatic invasion } & ly0 & 5 & ly1 & 6 \\
\hline & & & ly2 & 4 \\
\hline & & & ly3 & 0 \\
\hline & & $5(33 \%)$ & & $10(67 \%)$ \\
\hline
\end{tabular}

${ }^{a}$ Classification of Japanese Gastric Cancer Association

b Signet-ring cell carcinoma was included in poorly differentiated adenocarcinoma

metastastic node $(\mathrm{pN}+)$ by IHC staining were as follows: the tumor depth was SM2 or more in 11 (73\%), SM2 in 6, MP in 4, and SS in 1, and the histological type was moderately to poorly differentiated type in $12(80 \%)$, moderately in 6 , and poorly in 6 patients. Lymphatic invasion was positive in 10 patients $(75 \%)$, ly 1 in 6 , and ly 2 in 4 (Table 3). All 27 lymph nodes positive for ICG with infrared ray observation had micrometastasis or less (single cell 5, cluster 16, micrometastasis 6) (Table 4).

All but one patient $(0.8 \%)$ were recurrence-free with a follow-up period of 2-8 years (median 74.7 months) after surgery. The recurrent case was a 74-year-old patient with a T1N1 type 0-IIc signet cell tumor, $85 \mathrm{~mm}$ in diameter. This patient underwent total gastrectomy with free tumor margins and pick up of the SNs (Japanese node station numbers 3 and $4 \mathrm{~d}$ ). The frozen section of the SN was positive, and therefore D2 lymph node dissection was performed after the SN procedure. The final pathology report showed two metastatic nodes that were both SNs. This patient developed anastomosis recurrence 4.5 years after the first operation and died.
Table 4 Histological assessment of 27 IHC-positive but HE-negative lymph nodes

\begin{tabular}{lrll}
\hline & \multicolumn{2}{l}{ Lymphatic basins } \\
\cline { 2 - 3 } & \multicolumn{2}{l}{ Inside } & Outside \\
\cline { 2 - 3 } & ICG+ & ICG- & \\
\hline Isolated tumor cell $(<0.2 \mathrm{~mm})$ & & & \\
Single cell & 5 & 0 & 0 \\
Cluster & 16 & 0 & 0 \\
Micrometastasis $(0.2-2 \mathrm{~mm})$ & 6 & 0 & 0 \\
Macrometastasis $(>2 \mathrm{~mm})$ & 0 & 0 & 0 \\
\hline
\end{tabular}

\section{Discussion}

Kitagawa et al. [8] reported good outcomes of radioisotope (RI)-combined gastric cancer SNNS with SN identification $(35 / 36,97 \%)$, accurate diagnosis $(35 / 35,100 \%)$, and sensitivity $(5 / 5,100 \%)$. Hayashi et al. [13] achieved $100 \%$ identification and accurate diagnosis rates by a combination of RI and staining. We invented an infrared ray observation method using IREE to obtain a similar identification rate to RI-combined staining and reported good outcomes with $100 \%$ identification and accurate diagnosis and sensitivity [17]. Also in this study, the SN identification rate and sensitivity were both $100 \%$. Therefore, the infrared ray observation method was a useful method for detecting lymph node metastasis for standard gastrectomy and lymph node dissection.

To perform limited surgery associated with SNNS, accurate intraoperative diagnosis of lymph node metastasis is essential. In gastric cancer, Isozaki et al. [25] and Natsugoe et al. [26] reported that lymph node micrometastases were identified in 10-30\% of specimens by step sectioning or IHC. Our results were similar to these studies, and $13 \%$ of patients diagnosed with no metastasis by H\&E staining were judged lymph node positive by IHC staining. In breast cancer, $18 \%$ of patients diagnosed as having no SN metastasis by HE staining were diagnosed with micrometastasis by IHC staining, which failed to demonstrate any effect on prognosis during the mean observation period of 40 months [27]. Ishida et al. [28] reported that the presence of micrometastases in lymph nodes was indispensable in determining the prognosis of patients with gastric carcinoma. Saito et al. showed in 765 patients with early gastric cancer who were node negative by H\&E staining but had micrometastasis by IHC staining that 6 patients $(0.8 \%)$ exhibited recurrence of carcinoma. They advised that micrometastasis was an important prognostic factor and should be followed up for a long time [29]. Therefore, development of an accurate method for rapid intraoperative identification of metastatic lymph nodes in early gastric 
cancer is required. Matsumoto et al. [30] reported that rapid intraoperative immunostaining was a simple and useful technique for detecting lymph node micrometastasis. Yanagita et al. [22] showed that as a consequence of recent advances in RT-PCR technology, it now requires about 30-40 min to determine micrometastasis status. The combination of this method with SNNS may allow accurate intraoperative diagnosis of lymph node metastasis.

Kim et al. [31] indicated that lymphatic invasion and tumor size were important risk factors for micrometastasis. Our results also showed that micrometastasis was more frequent in patients with moderately to poorly differentiated adenocarcinoma with a tumor depth of SM2 or more and lymphatic invasion.

Ajisaka and Miwa [15] reported that lymph node metastasis was completely resected by LBD because all non-SN with micrometastasis by IHC staining and PCR assay was located in the same lymph node basin. Based on the above, Kitagawa et al. [19] proposed a combined method of LBD with wedge gastric resection or segmental gastrectomy in patients with no metastasis in the SN. Kinami et al. [23] established a dissection line in zones P, T, and D considering SN mapping. Also in this study, all lymph nodes diagnosed as metastatic by IHC staining but not by HE staining were classified as micrometastasis or less, and were detectable as ICG positive by the infrared ray method.

Lymph nodes with macrometastases can be diagnosed by intraoperative frozen section with $\mathrm{HE}$ staining, while micrometastases by $\mathrm{HE}$ staining alone are likely to be overlooked. Nevertheless, such lymph nodes can be completely removed by LBD. Therefore, ICG-positive lymphatic basins are first dissected by IREE, and the lymph nodes identified as SN are assessed by intraoperative frozen section diagnosis with $\mathrm{HE}$ staining. If the metastasis is found to be positive, D2 lymph node dissection is subsequently conducted. However, if the finding is negative, a limited surgery, such as wedge or segmental resection, can be applied. Micrometastasis can be completely removed by LBD, and an adequate effect of lymph node dissection is anticipated. This approach requires no intraoperative RTPCR diagnosis or IHC staining. However, it is absolutely necessary to carefully follow up such patients.

Conflict of interest We do not have any interest.

\section{References}

1. Maruyama K, Gunvén P, Okabayashi K, Sasako M, Kinoshita T. Lymph node metastases of gastric cancer: general pattern in 1931 patients. Ann Surg. 1989;210:596-602.

2. Nakajima T. Gastric cancer treatment guidelines in Japan. Gastric Cancer. 2002;5:1-5.
3. Gotoda T, Yanagisawa A, Sasako M, Ono H, Nakanishi Y, Shimoda T, Kato Y. Indication of lymph node metastasis from early gastric cancer: estimation with a large number of cases at two large centers. Gastric Cancer. 2000;3:219-25.

4. Seto Y, Shimoyama S, Kitayama J, Mafune K, Kaminishi M, Aikou T, Arai K, Ohta K, Nashimoto A, Honda I, Yamagishi H, Yamamura Y. Lymph node metastasis and preoperative diagnosis of depth of invasion in early gastric cancer. Gastric Cancer. 2001;4:34-8.

5. Gotoda T, Sasako M, Ono H, Katai H, Sano T, Shimoda T. Evaluation of the necessity for gastrectomy with lymph node dissection for patients with submucosal invasive gastric cancer. Br J Surg. 2001;88:444-9.

6. Morton DL, Wen DR, Wong JH, Economou JS, Cagle LA, Storm FK, Foshag LJ, Cochran AJ. Technical details of intraoperative lymphatic mapping for early stage melanoma. Arch Surg. 1992;127:392-9.

7. Giuliano AE, Kirgan DM, Guether JM, Morton DL. Lymphatic mapping and sentinel lymphadenectomy for breast cancer. Ann Surg. 1994;220:391-8.

8. Kitagawa Y, Fujii H, Mukai M, Ando N, Watanabe M, Ohgami M, Otani Y, Ozawa S, Hasegawa H, Furukawa T, Kumai K, Ikeda T, Nakahara T, Kubo A, Kitajima M. The role of the sentinel lymph node in gastrointestinal cancer. Surg Clin North Am. 2000;80:1799-809.

9. Hiratsuka M, Miyashiro I, Ishikawa O, Furukawa H, Motomura K, Ohigashi H, Kameyama M, Sasaki Y, Kabuto T, Ishiguro S, Imaoka S, Koyama H. Application of sentinel node biopsy to gastric cancer surgery. Surgery. 2001;129:335-40.

10. Kitagawa Y, Fujii H, Mukai M, Kubota T, Otani Y, Kitajima M. Radio-guided sentinel node detection for gastric cancer. $\mathrm{Br} \mathrm{J}$ Surg. 2002;89:604-8.

11. Miwa K, Kinami S, Taniguchi K, Fushida S, Fujimura T, Nonomura A. Mapping sentinel nodes in patients with early-stage gastric carcinoma. Br J Sug. 2003;90:178-82.

12. Ichikura T, Morita D, Uchida T, Okura E, Majima T, Ogawa T, Mochizuki H. Sentinel node concept in gastric carcinoma. World J Surg. 2002;26:318-22.

13. Hayashi H, Ochiai T, Mori M, Karube T, Suzuki T, Gunji Y, Hori S, Akutsu N, Matsubara H, Shimada H. Sentinel lymph node mapping for gastric cancer using a dual procedure with dye- and gamma probe-guided techniques. J Am Coll Surg. 2003;196:68-74.

14. Uenosono Y, Natsugoe S, Higashi H, Ehi K, Miyazono F, Ishigami S, Hokita S, Aikou T. Evaluation of colloid size for sentinel nodes detection using radioisotope in early gastric cancer. Cancer Lett. 2003;200:19-24.

15. Ajisaka H, Miwa K. Micrometastases in sentinel nodes of gastric cancer. Br J Cancer. 2003;89:676-80.

16. Kim MC, Kim HH, Jung GJ, Lee JH, Choi SR, Kang DY, Roh MS, Jeong JS. Lymphatic mapping and sentinel node biopsy using 99mTc-tin colloid in gastric cancer. Ann Surg. 2004;239:383-7.

17. Nimura H, Narimiya N, Mitsumori N, Yamazaki Y, Yanaga K, Urashima M. Infrared ray electronic endoscopy combined with indocyanine green injection of sentinel nodes of patients with gastric cancer. Br J Surg. 2004;91:575-9.

18. Isozaki H, Kimura T, Tanaka N, Satoh K, Matsumoto S, Ninomiya M, Ohsaki T, Mori M, Esophagus Gastrointestinal Surgical Treatment Study Group. An assessment of the feasibility of sentinel lymph node-guided surgery for gastric cancer. Gastric Cancer. 2004;7:149-53.

19. Kitagawa Y, Fujii H, Kumai K, Kubota T, Otani Y, Saikawa Y, Yoshida M, Kubo A, Kitajima M. Recent advances in sentinel node navigation for gastric cancer: a paradigm shift of surgical management. J Surg Oncol. 2005;90:147-52. 
20. Ohdaira H, Nimura H, Mitsumori N, Takahashi N, Kashiwagi H, Yanaga K. Validity of modified gastrectomy combined with sentinel node navigation surgery for early gastric cancer. Gastric Cancer. 2007;10:117-22.

21. Ishii K, Kinami S, Funaki K, Fujita H, Ninomiya I, Fushida S, Fujimura T, Nishimura G, Kayahara M. Detection of sentinel and non-sentinel lymph node micrometastases by complete serial sectioning and immunohistochemical analysis for gastric cancer. J Exp Clin Cancer Res. 2008;27:7.

22. Yanagita $S$, Natsugoe $S$, Uenosono $Y$, Arigami $T$, Arima $H$, Kozono T, Funasako Y, Ehi K, Nakajo A, Ishigami S, Aikou T. Detection of micrometastases in sentinel node navigation surgery for gastric cancer. Surg Oncol. 2008;17:203-10.

23. Kinami S, Fujimura T, Ojima E, Fushida S, Ojima T, Funaki H, Fujita H, Takamura H, Ninomiya I, Nishimura G, Kayahara M, Ohta T, Yoh Z. PTD classification: proposal for a new classification of gastric cancer location based on physiological lymphatic flow. Int J Clin Oncol. 2008;13:320-9.

24. Hermanek P, Hutter RVP, Sobin LH, Wittekind Ch. Classification of isolated tumor cells and micrometastasis. Cancer. 1999;86:2668-73.

25. Isozaki H, Okajima K, Fujii K. Histological evaluation of lymph node metastasis on serial sectioning in gastric cancer with radical lymphadenectomy. Hepatogastroenterology. 1997;44:1133-6.

26. Natsugoe S, Mueller J, Stein HJ, Feith M, Höfler H, Siewert JR. Micrometastasis and tumor cell microinvolvement of lymph nodes from esophageal squamous cell carcinoma: frequency, associated tumor characteristics, and impact on prognosis. Cancer. 1998;83:858-66.

27. Chagpar A, Middleton LP, Sahin AA, Meric-Bernstam F, Kuerer HM, Feig BW, Ross MI, Ames FC, Singletary SE, Buchholz TA, Valero V, Hunt KK. Clinical outcome of patients with lymph node negative breast carcinoma who have sentinel lymph node micrometastases detected by immunohistochemistry. Cancer. 2005;103:1581-6.

28. Ishida K, Katsuyama T, Sugiyama A, Kawasaki S. Immunohistochemical evaluation of lymph node micrometastases from gastric carcinomas. Cancer. 1997;79:1069-76.

29. Saito H, Osaki T, Murakami D, Sakamoto T, Kanaji S, Ohro S, Tatebe S, Tsujitani S, Ikeguchi M. Recurrence in early gastric cancer: presence of micrometastasis in lymph node of node negative early gastric cancer patients with recurrence. Hepatogastroenterology. 2007;54:620-4.

30. Matsumoto M, Natsugoe S, Ishigami S, Uenosono Y, Takao S, Aikou T. Rapid immunohistochemical detection of lymph node micrometastasis during operation for upper gastrointestinal carcinoma. Br J Surg. 2003;90:563-6.

31. Kim JJ, Song KY, Hur H, Hur JI, Park SM, Park CH. Lymph node micrometastasis in node negative early gastric cancer. Eur J Surg Oncol. 2008;35:409-14. 